\title{
Hernia Repair Via Laparoscopic Transabdominal Preperitoneal (TAPP) Method: Our Clinical Outcomes in County State Hospital
}

\section{Laparoskopik Transabdominal Preperitoneal (TAPP) Yöntem ile Fıtık Onarımı; İlçe Devlet Hastanesi Klinik Sonuçlarımız}

\author{
Ahmet Erdoğan, Ahmet Türkan, Uğur Kılınç, Mehmet Kağan Katar \\ Kahramanmaraş Elbistan Devlet Hastanesi, Genel Cerrahi Kliniği, Kahramanmaraş, Türkiye
}

Dergiye Ulaşma Tarihi:04.03.2018 Dergiye Kabul Tarihi:06.08.2018 Doi: 10.5505/aot.2018.63325

\begin{abstract}
ÖZET
GİRIŞ ve AMAÇ: İnguinal herni onarımı, genel cerrahide en sık yapılan ameliyatlardan birisidir. Çok sayıda yöntem tanımlanmıştır. Laparoskopik fitık onarımı 1990 'lı yıllardan sonra yapılmaya başlanmış ve birçok merkez tarafından benimsenmiştir. Çalışmamızın amacı, ilçe devlet hastanesinde yaptığımız laparoskopik transabdominal preperitoneal fitık onarımı sonuçlarımızı literatür eşliğinde değerlendirmektir. YÖNTEM ve GEREÇLER: Kasım 2015- Ağustos 2017 tarihleri arasında laparoskopik transabdominal preperitoneal yöntem ile inguinal herni onarımı yapılan hastaların sonuçları retrospektif olarak incelendi. BULGULAR: 30'u erkek, 3'ü kadın olmak üzere 33 hasta çalışmaya dahil edildi. Yaş ortanca değeri 46( 19 ile 75 arası) idi. $18(\% 54,6)$ hastada sağ, $4(\% 12,1)$ hastada sol ve $11(\% 33,3)$ hastada bilateral herni olmak üzere toplam 44 onarım yapıldı. Hastanede kalış süresi ortalama 1,09( $\pm 0,29)$ gün ve 1 ile 2 arasında idi. Ameliyat sonrası $2(\% 6,1)$ hastada psödonüks(seroma), $1(\% 3)$ hastada hematom, $2(\% 6,1)$ hastada orşit gözlendi. Hastaların takip süresi 6 ile 27 ay arası olup ortalama $13,76( \pm 5,84)$ idi. 33 hastanın takibinde $1(\% 3,1)$ hastada nüks tespit edildi.

TARTIŞMA ve SONUÇ: Laparoskopik fitık onarımının; küçük kesiler yapılması, daha erken iyileşme, daha az ağrı ve günlük aktiviteye erken dönüş sağlaması gibi avantajları olduğu bilinmektedir. Ancak öğrenme süreci açık yönteme göre daha uzundur. Uzmanlık eğitiminde veya daha sonraki süreçte laparoskopik yöntemin eğitimini alan kişilerde nüks oranında farklılık gözlenmediği bildirilmiş̧ir. Sonuç olarak laparoskopik yöntem eğitimini almış kişiler tarafından ilçe hastanelerinde de bu yöntemin güvenle yapılabileceği kanatindeyiz.
\end{abstract}

Anahtar Kelimeler: İnguinal Herni, Laparoskopi, Transabdominal Preperitoneal Onarım

\begin{abstract}
INTRODUCTION: Inguinal hernia repair is one of the most commonly performed operations in general surgery. A great number of methods has been described. Laparoscopic hernia repair was initiated to be performed after 1990s and adopted by many centers. The objective of this study is to evaluate our outcomes in laparoscopic transabdominal preperitoneal hernia repair which we performed in the county state hospital in the light of the literature. METHODS: Outcomes of patients for whom inguinal hernia repair was performed via laparoscopic transabdominal preperitoneal method between the dates of November 2015 and August 2017 were evaluated retrospectively.

RESULTS: 33 patients were included in the study, 30 being male and 3 being female. Median age value was 46 (ranging between 19 and 75). A total of 44 repairs were performed, being right-sided inguinal hernia in 18 $(54.6 \%)$ patients, left-sided in $4(12,1 \%)$ patients and bilateral in $11(33.3 \%)$ patients. Mean duration of hospital stay was $1,09( \pm 0,29)$ days and ranging between 1 and 2. Postoperatively, 2(6.1\%) patients were observed to develop pseudorecurrence (seroma), 1 (3\%) patient to develop hematoma and 2 (6.1\%)patients to develop orchitis. Mean follow-up duration of the patients was varying between 6 to 27 months, with a mean of 13,76( $\pm 5,84)$. During follow-up of 33 patients, $1(3,1 \%)$ patient was determined to develop recurrence.

DISCUSSION and CONCLUSION: Laparoscopic hernia repair has been known to have some advantages, including smaller incisions, more rapid recovery, less pain and earlier return to daily activities. However, process of learning takes longer compared to the open method. It was reported that no difference occurred in recurrence rates in individuals who received a training on the laparoscopic method during specialization training or during further educational processes. In conclusion, we considered that this method can also be safely performed in county hospitals by individuals who received training on the laparoscopic method.

Keywords: İnguinal Herni, Laparoskopi, Transabdominal Preperitoneal Onarım
\end{abstract}




\section{GİRiș}

İnguinal herni onarımı, genel cerrahide en s1k yapılan ameliyatlardan birisidir. Dünya genelinde y1llık 20 milyondan fazla herni onarımı yapıldığı tahmin edilmektedir[1]. İnguinal herni, üzerinde en çok tartışlan ve en fazla teknik tarif edilen konulardan bir tanesidir. İlk modern cerrahi tedavi tarifini 1884 yılında Bassini yapmıştır. Bassini tekniği, kişinin kendi dokusu kullanılarak yapılan ve uzun yıllar başarılı bir şekilde uygulanan bir tekniktir. Daha sonraları birçok teknik tarif edilmiştir[2]. 1980'lerde Lichtenstein'ın protez materyal kullanarak gerilimsiz anterior yaklaşımı yaygınlaştırması, doku onarımı ile yapılan tekniklerin kullanımını geride bırakmıştır[3]. İlk laparoskopik inguinal herni onarımı 1982 yılında Ger tarafindan uygulanmıştır. Ger, başka nedenler ile operasyonlar yaparken, herni tespit ettiği hastaların defektini de onarmıştır[4].

1990'lı yıllarda tarif edilen ve birçok fitık cerrahı tarafından tercih edilen transabdominal preperitonel (TAPP) ve total ektraperitoneal (TEP) fitık onarım yöntemleri en sık kullanılan laparoskopik yöntemlerdir[5]. TAPP yöntemi periton boşluğuna girilerek yapılır, alan k1sıtlaması yoktur, bunun için TEP'e göre daha az tecrübe gerektirir. TEP yönteminin ise, kısıtlı ve alışık olunmadık bir alanda yapıldığı için, daha fazla tecbüre gerektireceği ve TAPP'a göre kısmen daha zor bir teknik olduğu düşünülür. Ancak TEP'te karın boşluğuna girilmediği için; organ yaralanması, yapışıklık ve enfeksiyon gibi riskler TAPP'a göre daha azdir[1].

Bir ilçe devlet hastanesinde görev yapan genel cerrahi ekibi olarak günümüzdeki en modern tedavi tekniklerinden biri olan TAPP onarım tekniğini uygulamaktayız. İlçe devlet hastanesi olarak bu konudaki deneyim ve sonuçlarımızı literatür eşliğinde paylaşmak istedik.

\section{GEREÇ ve YÖNTEM}

Çalışmamız; Kahramanmaraş-Elbistan İlçe Devlet Hastanesinde Kasım 2015- Ağustos 2017 tarihleri arasında, üç genel cerrahi uzmanı tarafindan, laparoskopik TAPP yöntemi ile inguinal herni onarımı yapılan 33 hastanın dosya verilerine göre retrospektif olarak yapılmıştır.

Cerrahi teknik: Ameliyat öncesi tüm hastaların miksiyon yapması sağlandı ve intravenöz $1 \mathrm{gr}$ sefazolin ile proflaksi yapıldı. Ameliyatlar genel anestezi altında, supin ve $10^{0}-15^{0}$ trendelenburg pozisyonda gerçekleştirildi. Cerrah fitık alanının karşı tarafinda operasyonu yaparken cerrahın olduğu tarafta hastanın kolu kapatıld1. Göbek altından batına yerleştirilen $10 \mathrm{~mm}$ 'liktrokar ile $\mathrm{CO} 2$ insuflasyonu yapılarak pnömoperitoneum oluşturuldu. Bazı vakalarda $0^{0}$ ve bazılarında da $30^{\circ}$ kamera kullanıldı. Umbilikus seviyesinde sağ ve sol midklavikular hattan birer adet $5 \mathrm{~mm}$ 'lik trokarlar yerleştirildi. İnguinal bölgede periton açılarak bogros alanı, prevezikal alan ve erkekte spermatik yapilar ile kadında round ligament diseke edilerek her üç herni alanı ortaya kondu. Büyük herni keselerinde kese bir miktar diseke edildikten sonra bağlanarak kesildi. 10-12x15 cm boyutunda polipropilen mesh pubise, Cooper ligamanına ve batın ön duvarına emilebilir mesh sabitleyici ile tespit edildi. Bazı vakalarda periton süture edilerek bazılarında ise emilebilir mesh sabitleyici ile kapatılarak operasyon sonlandırıldi.

\section{BULGULAR}

Hastaların 30'u erkek, 3'ü kadın olarak izlendi. Yaş ortanca değeri 46, yaş aralı̆̆ 19 ile 75 arasında idi. 18(\%54,6) hastada sağ, 4(\%12,1) hastada sol ve 11(\%33,3) hastada bilateral herni olmak üzere toplam 44 onarım yapıldı. 1 hastaya aynı seansta kolesistektomi, 1 hastaya da aynı seansta böbrek kisti eksizyonu yapıldı. Hastaların 1(\%3)'i nüks herni, 32(\%97)'si primer herni idi. Onarımların 23(\% 52,3)'ü direkt herni, $19(\% 43,2)$ 'u indirekt herni ve 2'si $(\% 4,5)$ femoral herni idi. Herni tanis1 $18(\% 54,5)$ hastada preoperatif ultrason ile desteklenmişti, 15(\%44,5) hastanın ultrasonu yoktu. 1 hastada perioperatif kanama olduğu için açığa geçilerek anterior herniorafi yapıldı. Hastanede kalış süresi ortalama 1,09( $\pm 0,29)$ gün olarak saptand1. Ameliyat sonras1 2(\%6,1) hastada psödonüks, 1(\%3) hastada hematom, $2(\% 6,1)$ hastada orşit gözlendi. Hematom ve orşit olan hastalar nonoperatif olarak tedavi edildi. Hastaların takip süresi 6 ile 27 ay aras1 olup ortalama $13,76( \pm 5,84)$ idi. 33 hastanın 
takibinde $1(\% 3,1)$ hastada nüks tespit edildi (Tablo 1).

Tablo 1. Hastaların demografik özellikleri, fitık lokalizasyonu ve tipi, komplikasyon durumu, yapılan ek girişimler ve nüks durumu

\begin{tabular}{|c|c|c|c|}
\hline \multirow{3}{*}{ Cinsiyet } & & Sayı & $\%$ \\
\hline & Erkek & 30 & 91 \\
\hline & Kadın & 3 & 9 \\
\hline \multirow{3}{*}{$\begin{array}{c}\text { Fitık } \\
\text { Lokalizasyonu }\end{array}$} & Sağ & 18 & 54,6 \\
\hline & Sol & 4 & 12,1 \\
\hline & Bilateral & 11 & 33,3 \\
\hline \multirow{3}{*}{ Fitık Tipi } & Direkt & 23 & 52,3 \\
\hline & İndirekt & 19 & 43,2 \\
\hline & Femoral & 2 & 4,5 \\
\hline $\begin{array}{c}\text { Perioperatif } \\
\text { Komplikasyon }\end{array}$ & Kanama & 1 & 3 \\
\hline \multirow{3}{*}{$\begin{array}{c}\text { Postoperetif } \\
\text { Komplikasyon }\end{array}$} & $\begin{array}{l}\text { Pseudonüks } \\
\text { (Seroma) }\end{array}$ & 2 & 6,1 \\
\hline & Hematom & 1 & 3 \\
\hline & Orşit & 2 & 6,1 \\
\hline \multirow[b]{2}{*}{ Ek Girişim } & Kolesistektomi & 1 & \\
\hline & $\begin{array}{c}\text { Böbrek Kist } \\
\text { Eksizyonu }\end{array}$ & 1 & \\
\hline \multirow{2}{*}{ Fitık Oluşumu } & Primer & 32 & 97 \\
\hline & Nüks & 1 & 3 \\
\hline
\end{tabular}

\section{TARTIŞMA}

Laparoskopik cerrahi ilk uygulanmaya başlandığı günden bu yana ilgi odağı olmuş ve hızla birçok ameliyat için laparoskopik teknikler tanımlanmıştır. Cerrahi pratikte en sik uygulanan ameliyatlardan biri olan fittk cerrahisinde de başarılı bir şekilde uygulanmıştır[6].

Çeşitli teknikler tanımlansa da en sık TAPP ve TEP uygulanmaktadır. Bizim serimizde hastalara TAPP yöntemi uygulandi. Cerrahi ekibin TEP deneyimi olmasına rağmen TAPP onarımının tercih edilme nedeni, TEP yöntemi için balon trokar gibi ek malzemelere ihtiyaç olması ve ilçe devlet hastanesinde, bu ve benzeri malzemelerin temini için gerekli olan prosedürlerin aşılmasının zor olmasıdır.

Anterior yaklaşım sonrası oluşan nüks vakalarda, ameliyata sekonder doku değişikliklerinin posterior alanda beklenmemesi ve aynı trokar girişlerinden her iki inguinal alana hakim olunabilmesi nedeni ile nüks ve bilateral inguinal hernide laparoskopik onarım öncelikli olarak önerilmektedir[7]. Çalışmadaki olgularımızın 1'i nüks herni, 32'si primer herni nedeni ile opere edilirken, 11'inin de hernisi bilateral idi.
Laparoskopik yaklaşımın; küçük kesiler yapılması, daha erken iyileşme, ameliyat sonrası daha az ağrı, erken mobilizasyon ve günlük aktiviteye erken dönüş sağlamas1 gibi avantajları olduğu bilinmektedir. Bizim serimizde de hastanede yatış süresi ortalama 1,09 gün olup literatürle uyumlu bulunmuştur[8].

Laparoskopik onarım sırasında açık tekniğe dönülme oranı \%2-3 düzeylerinde bildirilmiştir[9, 10]. Bu çalışmada da literatürle uyumlu olarak 1 olguda intraoperatif kontrol edilemeyen kanama nedeni ile açık tekniğe dönülmüştür.

Seroma, hematom, testiste iskemi veya ağr1, hidrosel, epididimit, orşit ve kronik ağrı gibi komplikasyonlar görülebilmektedir [11, 12].

Çalışmamızda, ameliyat sonrası erken dönemde inguinal bölgede şişlik şikâyeti ve fitığın tekrarladığı endişesi ile başvuran 2 olguda seromaya bağlı psödonüks tespit edildi. Direkt herni nedeni ile onarım yapılan bu hastalarda, Reddy ve arkadaşlarının tarif ettiği gibi gevşek transvers fasya cebini inverte ederek cooper ligamanına fikse edilmemesinin psödonükse sebep olduğu düşüncesindeyiz [13].

Erken dönemde ağrı şikâyeti ile başvuran 1 hastanın yapılan USG'sinde, rektus kas1 posteriorunda ekstraperitoneal alanda hematom tespit edildi. Hematom tespit edilen olgu nonoperatif olarak takip edildi ve hematomun yaklaşık bir ayda spontan rezorbe olduğu görüldü. 2 olguda orşit görüldü. Orşit olan vakalar medikal tedavi ile düzeldi.

Herni onarımı için laparoskopik yaklaşım ile açık anterior yaklaşımın karşılaştırıldığ çalışmalarda nüks oranlarında farklılık görülmemiştir. Ancak açık cerrahi tekniklere göre laparoskopik tekniğin öğrenme süresinin daha uzun olduğu ve ögrenme sürecini tamamlamış deneyimli ellerde yapıldığında nüks oranlarının aynı olduğu da önemle vurgulanmıştır[6]. Matsumoto ve arkadaşlarının yaptığı bir çalışmada laparoskopik herni onarımında nükslerin beklenenden fazla görülmesi üzerine cerrahlara eğitim kursları verilmiş ve sonrasında nüks oranlarının beklenen seviyelere kadar gerilemiştir[14]. Wu ve arkadaşlarının yapmış olduğu meta-analizde TAPP tekniği uygulanmış 1336 hastada nüks oranı \%3,8 olarak bildirilmiş ve açı teknik ile 
karşılaştırıldığında nüks açısından anlamlı fark olmadığı tespit edilmiştir[5]. Bizim çalışmamızda da literatür ile uyumlu olarak $1(\% 3,1)$ hastada nüks gözlenmiştir.

Öğrenme sürecinin devam ettiği dönemde genç, zayıf, nüks olmayan erkek vakaların seçilmesi hem anatomik yapıların daha kolay seçilebilmesine hem de düşük nüks oranlarına katkı sağlayabilir[10, 15].

Sonuç olarak; yeterli sayıda laparoskopik fitık onarımı tecrübesi olan cerrahlar tarafından, ilçe hastanelerinde de laparoskopik fitık onarımının güvenle yapılabileceği kanısındayız. $\mathrm{Bu}$ konuda farklı merkezler tarafindan benzer çalışmalara gereksinim vardır.

Conflict of interest: The authors declare that they have no conflict of interest

\section{REFERENCES}

1. Wei FX, Zhang YC, Han W, Zhang Y, Shao Y, Ni R. Transabdominal preperitoneal (TAPP) versus totally extraperitoneal (TEP) for laparoscopic hernia repair: a meta-analysis. Surg Laparosc Endosc Percutan Tech. 2015;25:375-383.

2. Read RC. The development of inguinal herniorrhaphy. Surg Clin North Am. 1984;64:185196.

3. Shulman AG, Amid PK, Lichtenstein IL. A survey of non-expert surgeons using the open tension-free mesh patch repair for primary inguinal hernias. Int Surg. 1995;80:35-36.

4. Ger R. The management of certain abdominal herniae by intra-abdominal closure of the neck of the sac. Ann R Coll Surg Engl. 1982;64:342-344.

5. Wu JJ, Way JA, Eslick GD, Cox MR. Transabdominal Pre-Peritoneal Versus Open Repair for Primary Unilateral Inguinal Hernia: A Metaanalysis. World J Surg. 2017:1-8.

6. Cavazzola LT, Rosen MJ. Laparoscopic versus open inguinal hernia repair. Surg Clin North Am. 2013; 93:1269-1279.
7. Feliu X, Jaurrieta E, Vinas X, Macarulla E, Abad JM, Fernández-Sallent E. Recurrent inguinal hernia: a ten-year review. J Laparoendosc Adv Surg Tech A. 2004;14: 362-367.

8. Wauschkuhn CA, Schwarz J, Boekeler U, Bittner R. Laparoscopic inguinal hernia repair: gold standard in bilateral hernia repair? Results of more than 2800 patients in comparison to literature. Surg Endosc. 2010;24:3026-3030.

9. Swadia ND. Laparoscopic totally extra-peritoneal inguinal hernia repair: 9 year's experience. Hernia. 2011;15: 273-279.

10. Gökçal F, Çallı İ, Almalı N, Akkurt G. Laparoskopik Total Ekstraperitoneal Fitık Onarımı: İlk Deneyimlerimiz. Van Tip Dergisi. 2015;22: 256-259.

11. Dulucq JL, Wintringer P, Mahajna A. Laparoscopic totally extraperitoneal inguinal hernia repair: lessons learned from 3,100 hernia repairs over 15 years. Surg Endosc. 2009;23:482-486.

12. Dickinson KJ, Thomas M, Fawole AS, Lyndon PJ, White CM. Predicting chronic post-operative pain following laparoscopic inguinal hernia repair. Hernia. 2008;12: 597-601.

13. Reddy Reddy VM, Sutton CD, Bloxham L, Garcea G, Ubhi SS, Robertson GS. Laparoscopic repair of direct inguinal hernia: a new technique that reduces the development of postoperative seroma. Hernia. 2007;11:393-396.

14. Matsumoto $S$, Hayakawa $T$, Kawarada $Y$ et al. Proper training in laparoscopic hernia repair is necessary to minimize the rising recurrence rate in Japan. Asian journal of endoscopic surgery. DOI: 10.1111/ases.12433 Published Online 2017.

15. Schouten N, Elshof JWM, Simmermacher RKJ et al. Selecting patients during the "learning curve" of endoscopic Totally Extraperitoneal (TEP) hernia repair. Hernia. 2013;17: 737-743. 